\title{
The Effect of Hydrogenation on the Electrical Resistivity of Amorphous Alloys
}

\author{
M. ORNAT AND A. PAJA* \\ AGH University of Science and Technology, Faculty of Physics and Applied Computer Science, \\ Department of Solid State Physics, al. A. Mickiewicza 30, 30-059 Krakow, Poland
}

(Received May 6, 2014)

\begin{abstract}
Theoretical investigations of the influence of hydrogen contents on the electrical resistivity of amorphous metallic alloys have been carried out. We have made use of our method of calculations of the electrical resistivity of disordered systems based on the ground of Morgan-Howson-Saub and Evans models. Our method is fully quantum, includes multiple scattering effects and uses the scattering matrix operators for describing the electronion interactions. The model gives good agreement with experiment for many binary systems and should work for ternary systems as well, thus we performed calculations with hydrogen as one of the components of a ternary alloy. The results of our calculations show that the resistivity should increase with hydrogen concentration. Some experimental data confirm this predication.
\end{abstract}

DOI: 10.12693/APhysPolA.126.1296

PACS: 72.15.Cz, 72.15.Qm, 72.15.Rn

\section{Introduction}

Physical properties of amorphous metallic materials have been widely investigated by many authors since their obtainment by Klement et al. [1]. Their electron transport properties were the subject of special interest because of their extraordinary high resistivity and - very often - negative coefficient of resistivity which could give rise to a wide spectrum of applications (for an extensive review see e.g. $[2,3])$. In recent years a lot of materials - including amorphous ones - are examined as possible media for hydrogen storage [4]. Amorphous alloys are not so stable after hydrogenation as their polycrystalline counterparts. However, several authors reported fabrication of some stable phases of amorphous materials with hydrogen as one of the components so that the measurements of the resistivity became possible. Eliaz and Eliezer [5] examined various physical properties of ribbons of amorphous $\mathrm{Fe}_{80} \mathrm{~B}_{11} \mathrm{Si}_{9}$ alloy during hydrogenation and dehydrogenation. They found that the electrical resistivity was initially increasing upon hydrogen charging. Further behaviour of the resistivity depended on the current density but for high densities the increase continued. Azhazha et al. [6] measured the electrical resistivity of $\mathrm{Ti}_{66} \mathrm{Ni}_{20} \mathrm{Cu}_{10} \mathrm{Si}_{4}$ amorphous alloy doped with $6 \%$ (atomic) of hydrogen in the temperature range $4.2-$ $300 \mathrm{~K}$. They found that the resistivity decreased in the whole range of temperatures and the values of the resistivity of the sample doped with hydrogen were higher in that range. Narjis et al. [7-11] recently carried out extensive study of electron transport in hydrogenated amorphous silicon-nickel alloys. They thoroughly investigated the temperature and magnetic field dependence

*corresponding author; e-mail: paja@agh.edu.pl of the resistivity (conductivity) of those materials but not the concentration dependence. Therefore we undertook this task.

We have recently developed a method for calculation of the resistivity in disordered (liquid or amorphous) systems, obtaining very good results for many binary and ternary alloys. Here we present a proposition for calculation of the resistivity of hydrogen doped disordered systems.

In semi-classical Faber-Ziman (F-Z) model [12] liquid and amorphous alloys are considered as the sets of ions, relative positions of which are described with use of the partial structure factors, and the electron-ion interaction is represented by a pseudopotential. The model neglects quantum interference effects, which were shown to give significant influence on the resistivity, and the pseudopotential formalism makes the calculations extremely unstable [13]. F-Z model was extended by Evans et al. [14]. They replaced the pseudopotentials by the scattering matrix operators that were calculated basing on the phase shifts of the wave functions. Apart from improving the physical soundness of the results, another effects of the Evans correction are: stability of the results and reduction in number of model parameters. Another approach to the problem was presented by Morgan et al. [15], who based their investigations on quantum kinetic equation and obtained fully quantum model for resistivity, usually called the " $2 k_{\mathrm{F}}$ " scattering model (or MHŠ one). Their final formula for the resistivity may be interpreted as the rescaled $\mathrm{F}-\mathrm{Z}$ one, thus the improvement of the calculations by moving from the $\mathrm{F}-\mathrm{Z}$ model to the MHS one is simple and the results are much more physically sound. The difficulties here are created by the pseudopotentials that make the model unstable similarly to the $\mathrm{F}-\mathrm{Z}$ one.

We developed the MHS̆ model using the Evans correction to improve stability and reduce the number of parameters [16]. The Fermi energy has been calculated 
according to the Esposito [17] method, but we improved the phase-shifts calculations. In opposition to other authors we did not assume that the phase shift in zeroenergy limit is equal to zero and continuous for the whole energy range (or computed phase-shifts modulo $\pi$ ). We performed precise calculations, obtaining correct phase-shifts values, that allowed us to calculate effective valences of the elements basing on their number of electrons. Such method is more physically sound and eliminates one parameter (initial valence). Finally, we obtained the model with two parameters for each element (Slater exchange rate $\alpha$ and effective mass $m^{*}$ ) which were assumed to be fixed for each element and independent of the system the calculations were made for. Our method allowed us for successful calculations of the resistivity in binary and ternary alloys such as $\mathrm{Al}-\mathrm{Mg}, \mathrm{Cd}-\mathrm{Mg}, \mathrm{Zn}-\mathrm{Mg}, \mathrm{K}-\mathrm{Cs}, \mathrm{Na}-\mathrm{Cs}, \mathrm{Rb}-\mathrm{Cs}, \mathrm{K}-\mathrm{Rb}$, $\mathrm{Li}-\mathrm{Cd}, \mathrm{Ca}-\mathrm{Al}, \mathrm{K}-\mathrm{Rb}-\mathrm{Cs}, \mathrm{Mg}-\mathrm{Li}-\mathrm{Cd}$ with various concentrations of constituents.

Here we present exemplary results for the binary disordered system doped with hydrogen. In our approach the hydrogen is treated as the third element (except for the wave function which is analytical here for neutral atom) thus we can apply our method for ternary systems which turned out valid before. We did not focus here on a specific physically stable system but rather suggest the general method for studying the resistivity of such systems as a proposition for future calculations.

\section{Model}

The resistivity in the " $2 k_{\mathrm{F}}$ " scattering model is calculated as [15]:

$$
\rho=\frac{m_{\mathrm{e}}^{*}}{n e^{2}} \frac{1}{\tau_{\mathrm{FZ}}} \frac{1+\frac{1}{2} \frac{\tau_{\mathrm{FZ}}}{\tau} X^{2} F_{\mathrm{MHS}}^{1 / 2}(X)}{1-\frac{3}{64} X^{4} F_{\mathrm{MHS}}(X)},
$$

where $\tau_{\mathrm{FZ}}$ stands for the transport lifetime and $\tau$ is one-electron lifetime (both taken from the $\mathrm{F}-\mathrm{Z}$ model), $X=\hbar /\left(\tau E_{\mathrm{F}}\right)$, and

$$
F_{\mathrm{MHS}}(X)=\left[2 \ln \frac{z+1+2^{1 / 2}(z+1)^{1 / 2}}{z+1-2^{1 / 2}(z+1)^{1 / 2}}\right]^{2},
$$

where $z=\left(1+X^{2}\right)^{1 / 2}$. Instead of the original formula for $\mathrm{F}-\mathrm{Z}$ lifetimes we follow Evans [14] taking

$$
\begin{aligned}
& \frac{1}{\tau_{\mathrm{FZ}}}=\frac{m_{\mathrm{e}}^{*} \Omega_{0}}{4 \pi \hbar^{3} k_{\mathrm{F}}^{3}} \int_{0}^{2 k_{\mathrm{F}}} \mathrm{d} q \lambda(q) q^{3}, \\
& \frac{1}{\tau}=\frac{m_{\mathrm{e}}^{*} \Omega_{0}}{2 \pi \hbar^{3} k_{\mathrm{F}}} \int_{0}^{2 k_{\mathrm{F}}} \mathrm{d} q \lambda(q) q,
\end{aligned}
$$

where $\Omega_{0}$ is the average volume per one atom in the system, and for $p$-element alloy

$$
\lambda(q)=\sum_{\alpha=1}^{p} \sum_{\beta=1}^{p} \sqrt{c_{\alpha} c_{\beta}} S_{\alpha \beta}(q) t_{\alpha}(q) t_{\beta}^{*}(q),
$$

where $c_{i}$ stands for the number concentration of the $i$-th component, $S_{i j}$ are the Ashcroft-Langreth partial structure factors [18] and $t_{i}(q)$ are scattering operator matrix elements (at the Fermi level)

$$
t(q)=-\frac{2 \pi \hbar^{3}}{\Omega_{0} m_{\mathrm{e}}^{*} k_{\mathrm{F}}} \sum_{l}(2 l+1) \sin \eta_{l} \mathrm{e}^{\mathrm{i} \eta_{l}} P_{l}(\cos \theta),
$$

where $P_{l}$ are the Legendre polynomials, $\cos \theta=1-$ $q^{2} /\left(2 k_{\mathrm{F}}\right)^{2}$, and $\eta_{l}$ - phase shifts at the Fermi level for the $l^{\text {th }}$ band of the considered element.

\section{Results}

We performed numerical calculations for a model disordered system $\left(\mathrm{Li}_{1-x} \mathrm{Cd}_{x}\right)_{1-y} \mathrm{H}_{y}$. We treated it as a ternary system in our methodology. We took parameters $\alpha_{\mathrm{Li}}=0.75, m_{\mathrm{Li}}^{*}=1.16, \alpha_{\mathrm{Cd}}=1, m_{\mathrm{Cd}}^{*}=1.14$ and for hydrogen without any adjustments $\alpha_{\mathrm{H}}=1, m_{\mathrm{H}}^{*}=0.7$.

Figure 1 shows the electrical resistivity of amorphous alloys $\mathrm{Li}_{1-x} \mathrm{Cd}_{x}$ doped with hydrogen (crosses are experimental values for pure alloy after [19]). The solid line represents the relative resistivity $\rho_{y=0.1} / \rho_{y=0}$. In Fig. 2 we present the dependence of the resistivity on hydrogen concentration for several compositions of this alloy.

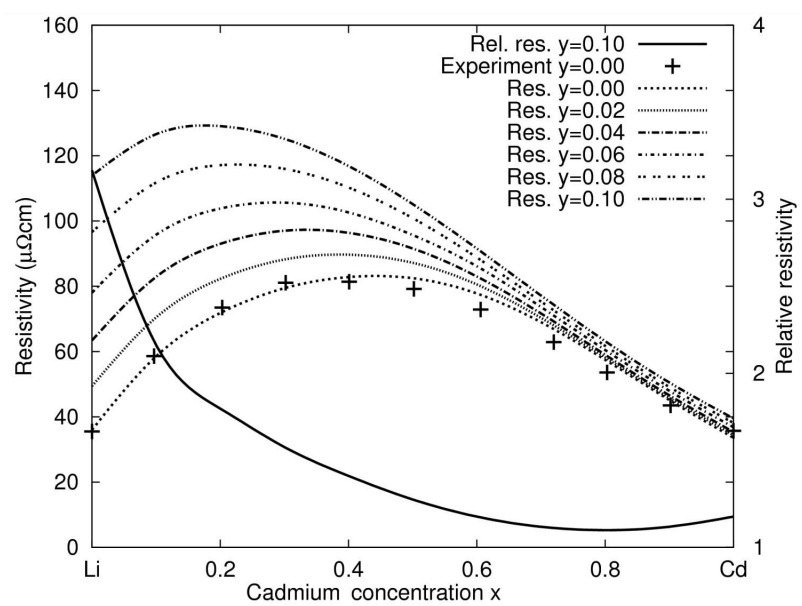

Fig. 1. Resistivity of $\left(\mathrm{Li}_{1-x} \mathrm{Cd}_{x}\right)_{1-y} \mathrm{H}_{y}$ as a function of cadmium number concentration $x$ for various hydrogen concentrations $y$ (crosses are experimental values after [19]). The solid line represents the relative resistivity $\rho_{y=0.1} / \rho_{y=0}$.

We can see in both figures that increasing the hydrogen contribution enhances the resistivity. The magnitude of the effect depends more on the element which is the component of the alloy than on the resistivity of the undoped alloy (in our case is the biggest for pure Li). The general trend obtained in our calculations i.e. the increase of the resistivity with hydrogen concentration is in agreement with the results of measurements reported in the works $[5,6]$.

\section{Summary}

We extended the MHS model for electron transport in disordered media by introducing the Evans correction and proposed the new method of calculation of the phase shifts. The model allows us for the calculation of the 


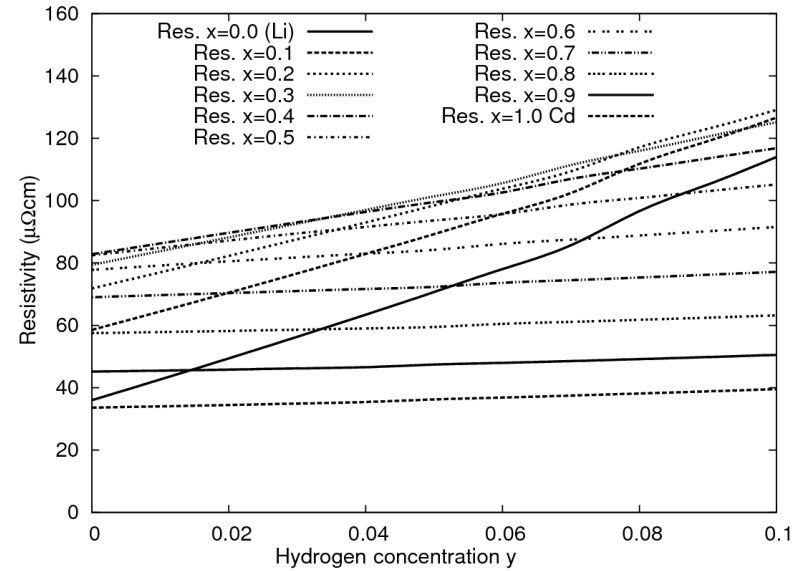

Fig. 2. Resistivity of $\left(\mathrm{Li}_{1-x} \mathrm{Cd}_{x}\right)_{1-y} \mathrm{H}_{y}$ as a function of hydrogen number concentration $y$ for several compositions of LiCd alloy.

resistivity of binary and ternary liquid and amorphous alloys with use of very limited number of parameters. We applied this model to amorphous alloys doped with hydrogen treating the hydrogen as a third component in a ternary alloy. Our results obtained for an exemplary amorphous alloy LiCd show that the resistivity of disordered systems should increase with hydrogen concentration. This expected trend agrees well with the measurements reported by other authors.

\section{Acknowledgments}

The work was partially supported by the Polish Ministry of Science and Higher Education and its grants for Scientific Research (grant number 11.11.220.01).

\section{References}

[1] W. Klement, R.H. Willens, P. Duwez, Nature 187, 869 (1960).

[2] Amorphous Metallic Alloys, Ed. F.E. Luborsky, Butterworths, London 1983.
[3] J.S. Dugdale, The Electrical Properties of Disordered Metals, Cambridge University Press, Cambridge (UK) 1995.

[4] Amorphous Materials: Research, Technology and Applications, Eds. J.R. Telle, N.A. Pearlstine, Nova Science Publishers, Hauppauge (USA) 2009.

[5] N. Eliaz, D. Eliezer, Metall. Mater. Trans. A $\mathbf{3 1 A}$, 2517 (2000).

[6] V. Azhazha, A. Grib, G. Khadzhay, B. Merisov, A. Pugachov, Int. J. Hydrogen Energy 28, 415 (2003).

[7] A. Narjis, A. El Kaaouachi, L. Limouny, S. Dlimi, A. Sybous, J. Hemine, R. Abdia, G. Biskupski, Physica B 406, 4155 (2011).

[8] A. Narjis, A. El Kaaouachi, S. Dlimi, A. Sybous, L. Limouny, R. Abdia, G. Biskupski, Moroccan J. Condens. Matter 13, 103 (2011).

[9] A. Narjis, A. El Kaaouachi, J. Hemine, A. Sybous, L. Limouny, S. Dlimi, R. Abdia, G. Biskupski, J. Mod. Phys. 3, 447 (2012).

[10] A. Narjis, A. El Kaaouachi, A. Sybous, L. Limouny, S. Dlimi, A. Aboudihab, J. Hemine, R. Abdia, G. Biskupski, J. Mod. Phys. 3, 517 (2012).

[11] A. Narjis, A. El Kaaouachi, S. Dlimi, A. Sybous, L. Limouny, G. Biskupski, Chin. J. Phys. 51, 593 (2013).

[12] T.E. Faber, J.M. Ziman, Philos. Mag. 11, 153 (1965).

[13] M. Ornat, A. Paja, J. Phys. Condens. Matter 20 , 375102 (2008).

[14] R. Evans, D.A. Greenwood, P. Lloyd, Phys. Lett. 35A, 57 (1971).

[15] G.J. Morgan, M.A. Howson, K. S̆aub, J. Phys. F Met. Phys. 15, 2157 (1985).

[16] M. Ornat, A. Paja, Appl. Phys. A 102, 379 (2011).

[17] E. Esposito, H. Ehrenreich, C.D. Gelatt, Phys. Rev. B 18, 3913 (1978).

[18] N.W. Ashcroft, D.C. Langreth, Phys. Rev. 159, 685 (1967).

[19] C. van der Marel, W. van der Lugt, J. Phys. F Met. Phys. 10, 1177 (1980). 